Pacific Journal of Mathematics

GROTHENDIECK AND WITT RINGS OF HERMITIAN FORMS 


\title{
GROTHENDIECK AND WITT RINGS OF HERMITIAN FORMS OVER DEDEKIND RINGS
}

\author{
Manfred Knebusch, Alex Rosenberg and Roger Ware
}

\begin{abstract}
The prime ideal theory of the Grothendieck and Witt ring of non-degenerate hermitian forms over a Dedekind ring with involution is studied. The relationship of these rings to those defined over the quotient field of the Dedekind ring is also investigated.
\end{abstract}

The main goal of this paper is to extend the structure theory for Witt rings over fields of Pfister [18] and Harrison-Leicht-Lorenz ([10], [16]) to the Grothendieck ring $K(C, J)$ and the Witt ring $W(C, J)$ of a Dedkind ring $C$ with an involution $J$. Since the case $J=$ identity is allowed, the Grothendieck and Witt rings of [12] are included. We shall see that the main theorems of Pfister and Harrison-LeichtLorenz remain true for $W(C, J)$ and that if $J$ is the identity they are also true for $K(C, J)$. However, for $K(C, J)$ with $J \neq$ identity there is some deviation: there may be $p$-torsion for primes $p \neq 2$ and there may be nilpotent elements which are not torsion (Example 1.3). This fact has been overlooked in [13].

In $\S 1$ we extend some elementary results of $[12, \S 11, \S 13]$ to the case $J \neq$ identity. We conjecture that they are well known to the specialists but we did not find an appropriate reference in the literature. We show that the canonical map from $W(C, J)$ to the Witt ring $W(L, J)$ of the quotient field $L$ of $C$ is injective and give some information about the kernel $\Lambda(C, J)$ of the map $K(C, J) \rightarrow$ $K(L, J)$. Since the exact determination of $\Lambda(C, J)$ is not needed for our structure theory we delay this matter to $\S 4$, where such a determination is given along the same lines as in [12, §11.2]. We then show that $W(C, J)$ is the intersection of certain subrings $W\left(C_{p}, J\right)$ of $W(L, J)$ which are Witt rings for abelian groups of exponent 2 in the sense of [14, Def. 3.12] and we describe the image $K^{\prime}(C, J)$ of $K(C, J)$ in $K(L, J)$ in an analogous way.

We are thus led to study subrings $T$ of an "abstract" Witt ring $R$ for an arbitrary abelian $q$-group [14, Def. 3.12]. If $T$ is the intersection of a family $\left\{T_{\alpha}\right\}$ of subrings of $R$ which are also Witt rings for some abelian $q$-groups, the entire prime ideal theory of $R$ remains true for $T$.

In $\S 3$ we show that if $T$ is either $K(C, J)$ or $W(C, J)$ then the group of units of $T$ is generated by $1+$ Nil $T$ and the rank one spaces

The second author was partially supported by NSF Grant GP-25600, and the third author was partially supported by NSF Grant GP-28915. 
over $(C, J)$.

The results of [14] are used throughout.

1. Elementary facts about $K(C, J)$ and $W(C, J)$. (cf. $[12, \S 11$, $\S 13.3]$, [9].)

In this paper $C$ will always denote a Dedekind ring and $J$ will be an involution on $C$ which is allowed to be the identity. The quotient field of $C$ will be denoted by $L$ and $J$ will also denote the unique extension of our involution to $L$. Further, $F$ denotes the fixed field under $J$ and $A$ denotes the intersection $F \cap C$. Since $C$ is integral over $A\left(c^{2}-(J(c)+c) c+J(c) c=0\right.$ for all $c$ in $\left.C\right), A$ is also a Dedekind ring and $C$ is the integral closure of $A$ in $L$. The unadorned $\otimes$ means $\otimes_{c}$.

Throughout we use the notations of [14]. In particular, we often write $\bar{x}$ for the value $J(x)$ of some $x$ in $L$ under $J$. A space $(E, \Phi)$, or more briefly $E$, over $(C, J)$ always means a fininitely generated projective $C$-module $E$ equipped with a non degenerate $J$-hermitian form $\Phi$ (see [14, Sec. 1], $\Phi$ is linear in the first argument and antilinear in the second). A space $E$ over $(C, J)$ is called metabolic if $E$ contains a direct summand $V$ as $C$-module with $V=V^{\perp}$ (see [14, Sec. 1]). By $S(C, J)$ we denote the semiring of isometry classes of spaces over $(C, J)$, by $K(C, J)$ we denote the corresponding Grothendieck ring, and by $W(C, J)$ the Witt ring of $(C, J)$, i.e., the residue class ring $K(C, J) / K M(C, J)$, where $K M(C, J)$ denotes the Grothendieck group of metabolic spaces over $(C, J)$ which is an ideal in $K(C, J)$ [14, Cor. 1.6]. We write $[E]$ for the image of the isometry class of a space $E$ in $K(C, J)$.

For any finitely generated projective $C$-module $U$ we denote by $U^{*}$ the group $\operatorname{Hom}_{C}(U, C)$ with $C$-module structure defined such that

$$
\left\langle u, c u^{*}\right\rangle=\bar{c}\left\langle u, u^{*}\right\rangle
$$

for all $u$ in $U, u^{*}$ in $U^{*}, c$ in $C$. We further denote by $H(U)$ the hyperbolic space $U \oplus U^{*}$ with the hermitian form $\Phi$ characterized by $\Phi(U \times U)=\Phi\left(U^{*} \times U^{*}\right)=0$ and $\Phi\left(u, u^{*}\right)=\left\langle u, u^{*}\right\rangle$ for $u$ in $U, u^{*}$ in $U^{*}$. The elements of $K M(C, J)$ all have the form $[H(U)]-[H(V)]$ ([14, Lemma 1.3(i)]). For the space $H(C)$ corresponding to the free $C$ module $C$ we simply write $H$. Analogous notations will be used over $(L, J)$.

Lemma 1.1. A space $E$ over $(C, J)$ is metabolic if the space $L \otimes E$ over $(L, J)$ is metabolic. Hence the canonical map from $W(C, J)$ to $W(L, J)$ induced by $E \mapsto L \otimes E$, is injective.

Proof. We repeat the well known argument for the convenience 
of the reader. Let $W$ be a subspace of $L \otimes E$ with $W^{\perp}=W$ and consider $E$ as a subset of $L \otimes E$. Then $V=W \cap E$ is a submodule of $E$ with $V=V^{\perp}$ and $V$ is also a direct summand of $E$ since $E / V$ is finitely generated and torsion free, hence projective [7, Prop. 4.1, p. 133]. The last assertion follows from the fact that over $(L, J)$ a space $M$ whose class in $W(L, J)$ is zero is metabolic $([3, \S 4]$ and in case of characteristic 2 and $J=$ identity, [12, Lemma 8.2.2, p. 119] or [17]).

We denote by $\Lambda(C, J)$ the kernel of the canonical map $[E] \mapsto[L \otimes E]$ from $K(C, J)$ to $K(L, J)$. Since the natural map $W(C, J) \rightarrow W(L, J)$ is injective it is clear that $\Lambda(C, J)$ is contained in $K M(C, J)$. Hence any element $x$ of $\Lambda(C, J)$ has the form

$$
x=[H(U)]-[H(V)]
$$

with $[H(U) \otimes L]=[H(V) \otimes L]$ in $K(L, J)$. Thus for some space $M$ over $(L, J)$ there is an isometry $M \perp(H(U) \otimes L) \cong M \perp(H(V) \otimes L)$ so that $\operatorname{rank} U=\operatorname{rank} V$. Conversely, it is evident that all such $x$ lie in $\Lambda(C, J)$. Now there exist ideals $\mathfrak{a}$ and $\mathfrak{b}$ of $C$ such that $U \cong \mathfrak{a} \oplus r \times C$ and $V \cong \mathfrak{b} \oplus r \times C$ for some $r \geqq 0$ where $r \times C$ denotes the free $C$ module of rank $r$ [22] or [20, Thm.1]. Hence by [14, Lemma 1.3(iii)],

$$
\left.x=[H(\mathfrak{a})]-[H(\mathfrak{b})]=[H(\mathfrak{a})]+\left[H(\mathfrak{b})^{-1}\right]-\left([H(\mathfrak{b})]+\left[H(\mathfrak{b})^{-1}\right)\right]\right) .
$$

Recalling that

$$
\mathfrak{a}_{1} \oplus \mathfrak{a}_{2} \cong C \oplus \mathfrak{a}_{1} a_{2}
$$

for any ideals $a_{1}, a_{2}$ of $C$ and again using Lemma 1.3(iii) of [14], we obtain $x=\left[H\left(\mathfrak{a b}^{-1}\right)\right]-[H]$.

We denote by $\operatorname{Pic}(C)$ the ideal class group of $C$, by $\operatorname{Pic}(C)^{G}$ the subgroup of all elements invariant under $G=\{1, J\}$, and by $(1+J) \operatorname{Pic}(C)$ the subgroup of all classes (a $\bar{a})$. Our consideration about $\Lambda(C, J)$ shows that we have a surjective map

$$
f: \operatorname{Pic}(C) \longrightarrow \Lambda(C, J)
$$

(cf. [12, 11.1.4, p. 136], [9, Th. 1]) which sends an ideal class (a) in $\operatorname{Pic}(C)$ onto $[H(\mathfrak{a})]-[H]$. By $\left(^{*}\right)$ we see that $f(\mathfrak{a} \mathfrak{b})=f(\mathfrak{a})+f(\mathfrak{b})$.

Proposition 1.2.

(i) $(1+J) \operatorname{Pic}(C) \subset \operatorname{Ker} f \subset \operatorname{Pic}(C)^{G}$.

(ii) If $J=$ identity then $2 \Lambda(C, J)=0$.

(iii) $\Lambda(C, J)^{2}=0$ (cf. [9, Prop. 5]).

Proof. (i) Since for any ideal $a$ of $C$ the $C$-modules $a^{*}$ and $\overline{\mathfrak{a}}^{-1}$ are isomorphic, we have $H(\mathfrak{a}) \cong H\left(\overline{\mathfrak{a}}^{-1}\right)$ and hence $f(\mathfrak{a})=f\left(\overline{\mathfrak{a}}^{-1}\right)$. Thus $f(\mathfrak{a} \bar{a})=0$ for all ideals $a$. On the other hand, if $\mathfrak{a}$ is an ideal 
with $f(\mathfrak{a})=0$ then there exists a space $E$ over $(C, J)$ with $H(\mathfrak{a}) \perp E \cong$ $H \perp E$. Comparing the Steinitz-invariants (= highest exterior powers) of both sides we see that $\left(a \bar{a}^{-1}\right)=1$ in $\operatorname{Pic}(C)$, hence $(\mathfrak{c})=(\bar{a})$.

Statement (ii) is now clear since $2 \operatorname{Pic}(C) \subset \operatorname{Ker} f$.

(iii) For two ideals $\mathfrak{a}, \mathfrak{b}$ we have

$$
H(\mathfrak{a}) \otimes H(\mathfrak{b}) \cong H\left(\mathfrak{a} \otimes\left(\mathfrak{b} \oplus \bar{b}^{-1}\right)\right) \cong H(\mathfrak{a b}) \perp H\left(a^{-1}\right)
$$

[14, Prop. 1.5 and Prop. 1.3]. From this one computes

$$
\begin{aligned}
f(\mathfrak{a}) f(\mathfrak{b}) & =[H(\mathfrak{a} \mathfrak{b})]+\left[H\left(\mathfrak{a} \overline{\mathfrak{b}}^{-1}\right)\right]-2[H(\mathfrak{a})]-2[H(\mathfrak{b})]+2[H] \\
& =f(\mathfrak{a} \mathfrak{b})+f\left(\mathfrak{a} \bar{b}^{-1}\right)-2 f(\mathfrak{a})-2 f(\mathfrak{b}) \\
& =f\left(\mathfrak{b}^{-1} \overline{\mathfrak{b}}^{-1}\right)=0 .
\end{aligned}
$$

EXAMPLE 1.3. Let $C$ be the ring of all integers of a quadratic number field with automorphism $J$. We have $A=\mathbb{Z}$, hence

$$
(1+J) \operatorname{Pic}(C)=0,
$$

so that $2 \operatorname{Pic}(C)^{\alpha}=0$. Hence $\Lambda(C, J)$ is a torsion group whose part prime to 2 equals the part prime to 2 of $\operatorname{Pic}(C)$. Thus we obtain many examples of Dedekind rings $(C, J)$ with $K(C, J)$ having odd torsion.

Furthermore, there are Dedekind rings $(C, J)$ such that $\Lambda(C, J)$ is not torsion at all. To obtain an example let $Y$ be an elliptic curve over the complex numbers and $X=Y-p$ for some point $p$ of $Y$. Since $X$ is a non singular affine curve its affine ring is a Dedekind ring $C$ with an involution $J$ induced by the inverse map $j$ of the abelian group $Y$ with unit $p$. There is a canonical isomorphism $\operatorname{Pic}(C) \cong Y$ which carries the induced action of $J$ on $\operatorname{Pic}(C)$ to $j$. Since as an abelian group $Y$ is $S^{1} \times S^{1}$, there are only four fixed elements in $Y$ and $Y$ is not torsion. This yields an example with $\Lambda(C, J)$ not torsion.

REMARK 1.4. In [9, Cor. to Th. 4] and [12, \$11] it has been shown that for $J=$ identity the kernel of $f$ is generated by $2 \operatorname{Pic}(C)$ and the classes of all maximal ideals $\mathfrak{P}$ with $2 \in \mathfrak{B}$. By the same method we show in $\S 4$ that for $J \neq$ identity, Ker $f$ is generated by the image of $\operatorname{Pic}(A)$ and all maximal ideals $\mathfrak{P}$ with $\mathfrak{P}^{2}=C(\mathfrak{P} \cap A)$.

For each maximal ideal $\mathfrak{p}$ of $A$ we set $C_{\mathfrak{p}}=A_{\mathfrak{p}} \otimes_{A} C=$ the localization of $C$ with respect to $\mathfrak{p}$, which is a semi-local Dedekind ring. The involution on $C_{\mathfrak{p}}$ induced by $J$ will also be denoted by $J$. We consider the $C_{p}$ as subrings of $L$ and have $C_{p}=A_{p} C$. Let $\operatorname{Im} S(C, J)$ and $\operatorname{Im} S\left(C_{p}, J\right)$ be the images of $S(C, J)$ and $S\left(C_{\Downarrow}, J\right)$ in $S(L, J)$.

Lemma 1.5. $\operatorname{Im} S(C, J)=\bigcap_{p} \operatorname{Im} S\left(C_{p}, J\right)$ where $\mathfrak{p}$ runs through the set, $\operatorname{Max} A$, of all maximal ideals of $A$. 
Proof. The assertion means that a space over $(L, J)$, which contains a $\left(C_{p}, J\right)$-space of full rank for all $\mathfrak{p}$ in $\operatorname{Max} A$, also contains a $(C, J)$-space of full rank. This is elementary lattice theory (cf. [6, Thm. 3, p. 54]).

By Lemma 1.1 we regard $W(C, J)$ and all $W\left(C_{p}, J\right)$ as subrings of $W(L, J)$. Since $\operatorname{Pic}\left(C_{\mathfrak{p}}\right)=0$ for each $\mathfrak{p}$ and hence by Proposition 1.2, $\Lambda\left(C_{p}, J\right)=0$, we similarly regard the rings $K\left(C_{p}, J\right)$ as subrings of $K(L, J)$. We denote by $K^{\prime}(C, J)$ the image of $K(C, J)$ in $K(L, J)$.

LEMMA 1.6.

(i) $K^{\prime}(C, J)=\bigcap_{\mathfrak{p}} K\left(C_{p}, J\right)$

(ii) $W(C, J)=\bigcap_{\mathfrak{p}} W\left(C_{\mathfrak{p}}, J\right)$

where in both equations $\mathfrak{p}$ runs through the maximal ideals of $A$.

Proof. Since all projective modules over $C_{p}$ are free, $K M(L, J)$ and all $K M\left(C_{p}, J\right)$ coincide with the additive group generated by $[H]$. Evidently $K M(C, J)$ also maps onto $K M(L, J)$. Thus as subsets of $W(L, J)$ we get $W\left(C_{p}, J\right)=K\left(C_{p}, J\right) / K M(L, J)$ and

$$
W(C, J)=[K(C, J) / \Lambda(C, J)] /[K M(C, J) / \Lambda(C, J)]=K^{\prime}(C, J) / K M(L, J)
$$

so that the two assertions of the lemma are equivalent.

We proceed to prove (ii). Let $x$ be an element of $\cap W\left(C_{p}, J\right)$ and $(V, \Phi)$ a space over $(L, J)$ representing $x$. As for any $(L, J)$-space there exists a finite "exceptional set" $T \subset \operatorname{Max} A$ such that $V$ contains a space over $\left(C_{p}, J\right)$ of full rank for all $\mathfrak{p} \notin T$. If $T \neq \varnothing$ let $\mathfrak{p}_{0}$ be a prime ideal in $T$. Since the class of $V$ lies in $W\left(C_{p_{0}}, J\right)$ there exists a space $V_{0}$ over $(L, J)$ containing a $\left(C_{p_{0}}, J\right)$-space of full rank and metabolic $(L, J)$-spaces $U, U_{0}$ such that $V \perp U \cong V_{0} \perp U_{0}$ [14, Lemma 1.4]. Since any metabolic space over $(L, J)$ contains $\left(C_{\mathfrak{p}}, J\right)$-spaces of full rank for all $\mathfrak{p}$ in $\operatorname{Max} A$ (see the definition of metabolic spaces in [14, Sec. 1]) it follows that $V \perp U$ is a space over $(L, J)$ which represents $x$ and has an exceptional set contained in $T-\left\{\mathfrak{p}_{p_{0}}\right\}$. After a finite number of such steps we obtain a space $\widetilde{V}$ representing $x$ and containing $\left(C_{\mathfrak{p}}, J\right)$-spaces of full rank for all $\mathfrak{p}$. Hence by Lemma 1.5 $\widetilde{V}$ contains a $(C, J)$-space of full rank. Thus $\bigcap W\left(C_{p}, J\right) \subset W(C, J)$. The reverse inclusion is clear.

REMARK. Using the cancellation theorem for hermitian forms over fields [3, Thm. 1, p. 71], Hilfsatz 13.3.3 of [12] can be extended to the case $J \neq$ identity, stating that if a space $V$ over $(L, J)$ contains a $\left(C_{p}, J\right)$-space of full rank then the same is true for all spaces $V^{\prime}$ with the same class in $W(L, J)$. This offers a shorter proof of Lemma 1.6(ii).

For any $\mathfrak{p}$ in $\operatorname{Max} A$ we have either $\mathfrak{p} C=\mathfrak{P}$ or $=\mathfrak{P}^{2}$ or $=\mathfrak{P} \overline{\mathfrak{P}}, \mathfrak{P} \neq$ 
$\overline{\mathfrak{P}}$, with $\mathfrak{P}$ in $\operatorname{Max} C$ [5, Th. 2, p. 42].

Lemma 1.7. If $\mathfrak{p} C=\mathfrak{P} \overline{\mathfrak{P}}$ with $\mathfrak{P} \neq \overline{\mathfrak{P}}$ or $\mathfrak{p} C=\mathfrak{P}^{2}$ then $K\left(C_{p}, J\right)=$ $K(L, J)$ and $W\left(C_{p}, J\right)=W(L, J)$.

Proof. We denote the norm function $x \mapsto x \bar{x}$ ( $x \mapsto x^{2}$ if $J=$ identity) by $N$. Since $K(L, J)$ and $W(L, J)$ are both generated by one dimensional forms it suffices to show that any coset of $F^{*} \bmod N L^{*}$ contains a unit of $C_{p}$. Now, the coset of an element $x$ of $F^{*} \bmod N L^{*}$ contains a unit of $C_{p}$ if and only if the coset of $x$ in $\hat{F}_{p}^{*} \bmod N \hat{L}_{p}^{*}$ contains a unit of $\hat{C}_{\mathfrak{p}}\left(\hat{F}_{\mathrm{p}}, \hat{L}_{\mathfrak{p}}, \hat{C}_{\mathrm{p}}, \cdots\right.$ denote the completions of $F, L$, $C, \cdots$ with respect to $\mathfrak{p})$. Evidently this is true if $\mathfrak{p} C=\mathfrak{P}^{2}$. If $\mathfrak{p} C=$ $\mathfrak{P} \overline{\mathfrak{P}}$ with $\mathfrak{P} \neq \overline{\mathfrak{F}}$ then $\hat{L}_{\mathfrak{p}}=\hat{F}_{\mathfrak{p}} \times \hat{F}_{\mathfrak{p}}$ and the involution of $\hat{L}_{\mathfrak{p}}$ corresponds to the involution $(a, b) \mapsto(b, a)$ of $\hat{F}_{p} \times \hat{F}_{p}[14$, Example 1.7]. Thus $N\left(\hat{L}_{p}^{*}\right)=\hat{F}_{p}^{*}$ and the assertion is also true in this case.

REMARK 1.8. If $\mathfrak{p} C=\mathfrak{P}$ then $K\left(C_{p}, J\right) \neq K(L, J)$ and $W\left(C_{p}, J\right) \neq$ $W(L, J)$. In fact, one has an exact sequence

$$
O \longrightarrow W\left(C_{\mathfrak{p}}, J\right) \longrightarrow W(L, J) \stackrel{\partial_{\mathfrak{p}}}{\longrightarrow} W(C / \mathfrak{P}, J) \longrightarrow 0
$$

with residue class form homomorphism $\partial_{p}$ (cf. [21], [12, §13.3], [19]).

Combining Lemmas 1.6 and 1.7 we get

Proposition 1.9 .

(i) $K^{\prime}(C, J)=\bigcap_{p} K\left(C_{p}, J\right)$

(ii) $W(C, J)=\bigcap_{p} W\left(C_{p}, J\right)$

where in both equations $\mathfrak{p}$ runs through all maximal ideals of $A$ with $\mathfrak{p} C$ a maximal ideal of $C$.

2. Subrings of Witt rings. Let $q$ be a rational prime and $G$ an abelian $q$-group. In [14, Def. 3.12] we have called a commutative ring $R$ a Witt ring for $G$ if $R$ is a homomorphic image of the integral group ring $Z[G]$ and the torsion subgroup, $R_{t}$, of $R$ is $q$-primary. Let us recall some facts proved for a Witt ring $R$ in [14]: There is only one prime ideal $M_{0}$ of $R$ which contains $q$. The ideal $M_{0}$ is of finite index $q$ (i.e., $R / M_{0} \cong \boldsymbol{F}_{q}^{1)}$ ) and contains all minimal prime ideals of $R$. Moreover, any maximal ideal $M \neq M_{0}$ of $R$ properly contains a unique prime ideal and this prime ideal is a minimal prime ideal. All the zero divisors of $R$ lie in $M_{0}$, the ring $R$ is connected (i.e., has no idempotents other than 0 or 1 ) and $R_{t} \neq 0$ if and only if $M_{0}$ consists entirely of zero divisors. $R$ is integral over $Z$ and hence $R$ is a Jacobson ring [5, p. 67] of Krull dimension, $\operatorname{dim} R \leqq 1$. In particular, its Jacobson radical, Rad $R$, coincides with its nil radical,

\footnotetext{
${ }^{1} \boldsymbol{F}_{q}$ denotes the field of $q$-elements.
} 
Nil $R$. In addition, $R_{t}=$ Nil $R$ if and only if $Z \rightarrow R$ is injective and $R_{t}=R$ otherwise. In the former case the minimal prime ideals $P$ of $R$ are characterized by $P \cap Z=0$ and in the latter case $R$ is local and $M_{0}$ is the only prime ideal of $R$.

Let $C$ be a Dedekind ring with involution $J$ and quotient field $L$. It is pointed out in [14, Remark 3.11] that $K(L, J)$ and $W(L, J)$ are Witt rings for an abelian group of exponent two. By Lemma 1.1, $W(C, J)$ is a subring of $W(L, J)$ and by Proposition 1.2 the natural map $K(C, J) \rightarrow K(L, J)$ has a kernel $\Lambda(C, J)$ which is small in the sense then $\Lambda(C, J)^{2}=0$, and if $J$ is the identity, in addition $2 \Lambda(C, J)=0$. We are thus led to consider subrings $T$ of a Witt ring $R$ for an abelian $q$-group.

The following Lemma follows easily from the properties of $R$ described above, the Lying over theorem [5, Th. 1, p. 38], and [14, Lemma 2.5]. Its proof will be omitted.

Lemma 2.1. Let $T$ be a subring of a Witt ring $R$ for an abelian q-group. Then

(i) $T$ is integral over $Z, T$ is a Jacobson ring, and $T$ is connected.

(ii) $T$ has a unique prime ideal $M_{0, T}$ containing $q$ and $T / M_{0, T} \cong \boldsymbol{F}_{q}$.

(iii) If $M_{0, T}$ is not the only prime ideal of $T$ then every maximal ideal properly contains a prime ideal. In this case $Z \rightarrow T$ is injective and a prime ideal $P$ is minimal if and only if $P \cap \boldsymbol{Z}=0$.

(iv) $T=T_{t}$ if and only if $M_{0, T}$ is the only prime ideal of $T$.

(v) $T_{t}$ is q-primary.

(vi) All zero divisors of $T$ lie in $M_{0, T}$ and $M_{0, T}$ consists entirely of zero divisors if and only if $T_{t} \neq 0$.

(vii) If $T_{t} \neq T$ then Nil $T=T_{t}$.

In particular, the statements of the lemma are true for $W(C, J)$ and $K^{\prime}(C, J)$. Because of the stated properties of $\Lambda(C, J)$ they also remain true for $K(C, J)$ if $J$ is the identity. If $J$ is not the identity (i), (ii), and (iii) remain true for $K(C, J)$ and (iv) is vacuous because $K(C, J)$ always contains $Z$. As shown by Example 1.3, (v), (vi), and (vii) are generally not true. However, even in this case $K(C, J)_{t} \subset$ Nil $K(C, J)$ since $\Lambda(C, J)^{2}=0$ and $K^{\prime}(C, J)_{t} \subset$ Nil $K^{\prime}(C, J)$.

REMARK 2.2. Suppose $G$ is group of exponent two and $Z \rightarrow T$ is injective. If $P$ is a minimal prime ideal of $T$ there is a minimal prime ideal $P^{\prime}$ of $R$ such that $P^{\prime} \cap T=P$ [11, Ex. 1, p. 41]. Moreover, by [14, Remark 3.2], $R / P^{\prime} \cong Z$ so $T / P$ is a subring of $Z$ and hence $T / P \cong Z$. Thus we see that any homomorphism from $T$ to $Z$ extends to one from $R$ to $Z$ and Nil $T$ is the intersection of the 
kernels of the homomorphisms from $T$ to $\mathcal{Z}$. Moreover, if $M$ is a maximal ideal of $T$ we must have $T / M \cong \boldsymbol{F}_{p}$ for some rational prime $p$. Thus if $C$ is a Dedekind ring with involution $J$ and $P$ is a non maximal, whence minimal, prime ideal of $T=K(C, J)$ or $W(C, J)$ then $T / P \cong Z$. If $M$ is a maximal ideal of $T=K(C, J)$ or $W(C, J)$ then $T / M \cong \boldsymbol{F}_{p}$ for some rational prime $p$. Moreover, in these cases the ideal $M_{0, T}$ is exactly the ideal of forms of even rank.

At the beginning of this section we stated one property of Witt rings $R$ which is not necessarily inherited by all subrings of $R$ : a maximal ideal $M \neq M_{0}$ contains a unique minimal prime ideal. We obtained this property in [14] as a consequence of the following theorem: If $\widetilde{R}$ denotes the integral closure of the image $\bar{R}=1 \otimes R$ of $R$ in $Q \otimes R$ then $\widetilde{R} / \bar{R}$ is a $q$-group. Here, and until the end of $\S 2$, $\otimes$ always denotes $\otimes_{z}$. This results from the rather evident fact, that $R / p R$ is von Neumann regular for all $p \neq q$ (see [14]). Thus we are now looking for subrings $T$ of $R$ such that $T / p T$ is von Neumann regular for all $p \neq q$. For any such subring it will be true that a maximal ideal $M \neq M_{0, T}$ contains a unique minimal prime ideal.

For any abelian group $X$ and rational prime $p$ we let $X[p]$ be the $p$-primary component of $X$.

Lemma 2.3. Let $T \subset R$ be an integral extension of commutative rings and $p$ a rational prime such that $R[p]=0$ and $R / p R$ is von Neumann regular. Then $T / p T$ is von Neumann regular if and only if $(R / T)[p]=0$.

Proof. By [14, Lemma 2.8], Nil $(T / p T)=0$ if and only if $(R / T)[p]=0$. Now by Lemma 2.7 of [14] the kernel of the map $T / p T \rightarrow R / p R$ is a nil ideal. Since $R / p R$ is integral over $T / p T$, $\operatorname{dim} T / p T=\operatorname{dim} R / p R=0$. Thus $T / p T$ is von Neumann regular if and only if $\mathrm{Nil}(T / p T)=0$ [4, Ex. 16(d), p. 173], which proves the lemma.

COROLlaRy 2.4. Let $R$ be a commutaive ring and $\left\{T_{i}\right\}_{i \in I}$ a family of subrings such that $R$ is integral over $T=\bigcap_{i \in I} T_{i}$. If for a rational prime $p$ with $R[p]=0$ all the rings $R / p R, T_{i} / p T_{i}$ are von Neumann regular then $T / p T$ is also von Neumann regular.

Proof. Consider the exact sequence

$$
0 \longrightarrow R / T \longrightarrow \prod_{i \in 1} R / T_{i}
$$

of abelian groups. By Lemma 2.3, we have $\left(R / T_{i}\right)[p]=0$ for all $i$ so $(R / T)[p]=0$. Hence Lemma 2.3 shows that $T / p T$ is von Neumann regular. 
THEOREM 2.5. Let $R$ be a Witt ring for an abelian q-group $G$ and let $\left\{T_{i}\right\}_{i \in I}$ be a family of subrings of $R$ such that each $T_{i}$ is a Witt ring for an abelian q-group $H_{i}$. If $T=\bigcap_{i \in I} T_{i}$ then

(i) $T / p T$ is von Neumann regular for all rational primes $p \neq q$.

(ii) If $\widetilde{T}$ denotes the integral closure of $\bar{T}=1 \otimes T$ in $Q \otimes T$ then $\widetilde{T} / \bar{T}$ is a q-group.

(iii) Any maximal ideal of $T$ distinct from $M_{0, T}$ properly contains a unique prime ideal of $T$ which is a minimal prime ideal.

Proof. Since $R_{t}$ is $q$-primary, $R[p]=0$ for all rational primes $p \neq q$. Moreover, by [14, Example 2.6] $R / p R$ and $T_{i} / p T_{i}$ are von Neumann regular rings. Statement (i) now follows immediately from Corollary 2.4. Statement (ii) is then a consequence of [14, Prop. 2.9]. Finally, if $T$ contains a maximal ideal distinct from $M_{0, T}$ then by Lemma 2.1 (iv) and (vii), Nil $T=T_{t}$. Thus (iii) follows from (i) and [14, Cor. 2.10 and Th. 2.12].

EXAMPLES 2.6. (i) Let $G$ be an abelian $q$-group and $\left\{H_{i}\right\}_{i \in I}$ a family of subgroups of $G$. Let $K$ be an ideal of $Z[G], R=Z[G] / K$, and for $i$ in $I$, let $T_{i}=Z\left[H_{i}\right] /\left(Z\left[H_{i}\right] \cap K\right)$. If $R$ is a Witt ring for $G$ then each $T_{i}$ has only $q$-torsion and so is a Witt ring for $H_{i}$. Hence $T=\bigcap T_{i}$ satisfies the conclusions of Theorem 2.5.

(ii) Let $C$ be a Dedekind ring with involution $J$ and quotient field $L$. Denote the fixed ring of $J$ on $C$ by $A$. By Proposition 1.9,

$$
K^{\prime}(C, J)=\bigcap_{p} K\left(C_{p}, J\right), \quad W(C, J)=\bigcap_{p} W\left(C_{p}, J\right)
$$

where $\mathfrak{p}$ runs through all maximal ideals of $A$ with $\mathfrak{p} C$ a maximal ideal of $C$. Since each $C_{p}$ is a local ring it follows that $K\left(C_{p}, J\right)$ and $W\left(C_{p}, J\right)$ are Witt rings for an abelian group of exponent two [14, Remark 3.11]. Hence the conclusions of Theorem 2.5 apply to $K^{\prime}(C, J)$ and $W(C, J)$. Since by Proposition 1.2(iii) the kernel $\Lambda(C, J)$ of $K(C, J) \rightarrow K(L, J)$ is nilpotent, statement (iii) of Theorem 2.5 remains true for $K(C, J)$. In case $J$ is the identity, Proposition 1.2(ii) asserts that $2 \Lambda(C, J)=0$. Hence $\boldsymbol{Q} \otimes K(C, I d)=\boldsymbol{Q} \otimes K^{\prime}(C, I d)$ and

$$
K(C, I d) / p K(C, I d) \cong K^{\prime}(C, I d) / p K^{\prime}(C, I d)
$$

for all odd $p$ so that statements (i) and (ii) of Theorem 2.5 hold for $K(C, I d)$ also.

We finally remark that a shorter proof of Theorem 2.5(ii) and hence, using [14, Cor. 2.10 and Th. 2.12], also of Theorem 2.5(iii) can be given by way of

LEMma 2.7. Let $R$ be a commutative ring, $\left\{T_{i}\right\}_{i \in I}$ a family of 
subrings and $T=\bigcap_{i \in I} T_{i}$. Let $p$ be a rational prime such that $R[p]=\left(\widetilde{T}_{i} / \bar{T}_{i}\right)[p]=0$ for all $i$ in $I$. Then $(\widetilde{T} / \bar{T})[p]=0$.

Proof. Since $\boldsymbol{Q}$ is a flat $\boldsymbol{Z}$-module we may identify $\boldsymbol{Q} \otimes T_{i}$ and $\boldsymbol{Q} \otimes T$ with subrings of $\boldsymbol{Q} \otimes R$. Thus we regard $\bar{T}_{i}, \widetilde{T}_{i}, \bar{T}$, and $\widetilde{T}$ as subrings of $\boldsymbol{Q} \otimes R$. Let $\widetilde{x}$ be an element of $\widetilde{T}$ with $p \widetilde{x}$ in $\bar{T}$. Then for all $i$ in $I$ we have $\widetilde{x} \in \widetilde{T}_{i}$ and $p \widetilde{x} \in \bar{T}_{i}$. Hence $\widetilde{x} \in \bigcap_{i \in I} \bar{T}_{i}$. There thus exist elements $x_{i}$ in $T_{i}$ and $y$ in $T$ such that $\widetilde{x}=1 \otimes x_{i}$ and $p \widetilde{x}=1 \otimes y$. Hence $p x_{i}-y$ lies in $R_{t}$ for all $i$ in $I$. Thus there exist integers $n_{i}$ with $\left(n_{i}, p\right)=1$ such that $n_{i} p x_{i}=n_{i} y$. Now there are integers $a_{i}, b_{i}$ with $1=a_{i} n_{i}+b_{i} p$. Multiplying this last relation by $y$ yields elements $z_{i}$ in $T_{i}$ with $y=p z_{i}$ for all $i$ in $I$. But since $R[p]=0$ the relation $p z_{i}=p z_{j}$ shows $z_{i}=z_{j}$ for all $i, j$ in $I$. Hence there is an element $z$ in $T$ with $y=p z$. Thus $p \tilde{x}=1 \otimes p z$ and therefore $\widetilde{x}=1 \otimes z$ which shows that $\widetilde{x}$ lies in $\bar{T}$, i.e. $(\widetilde{T} / \bar{T})[p]=0$.

3. Units in $K(C, J)$ or $W(C, J)$. We first recall some elementary facts true for spaces over an arbitrary commutative ring $C$ with involution $J$. For any space $(E, \Phi)$ over $(C, J)$ there is an inverse space $(\hat{E}, \widehat{\Phi})$ [3, Def. 8, p. 23] defined as follows: $\hat{E}$ is a $C$-module on the additive group $E$ with a new scalar multiplication $c \cdot x=J(c) x$ and the form $\hat{\Phi}$ on $\hat{E}$ is defined by $\widehat{\Phi}(x, y)=\Phi(y, x)$. Now assume that $E$ has rank one. Then it is easily checked that the $C$-linear map $g: E \otimes$ $\widetilde{E} \rightarrow C$ defined by $g(x \otimes y)=\Phi(x, y)$ is bijective and gives an isometry from $(E \otimes \widetilde{E}, \Phi \otimes \widehat{\Phi})$ to the space $(C, \Omega)$ where $\Omega(c, d)=c \bar{d}$ for $c, d$ in $C$ (by a localization argument it suffices to check this for $E$ a free space in which case the proof is straightforward). Since the space $(C, \Omega)$ has matrix (1) it is clear that the tensor product makes the set $S_{1}(C, J)$ of isometry classes of $(C, J)$-spaces of rank one into an abelian group. The subgroup of classes of free spaces of rank one can be identified with $A^{*} / N C^{*}$ where $A$ denotes the fixed ring of $J$ and $C^{*}, A^{*}$ are the groups of units of $C, A$.

REMARK 3.1. On the group Pic $(C)$ of projective $C$-modules of rank one, $J$ induces an involution $(M) \mapsto(\widehat{M})$, where again $\hat{M}$ denotes the abelian group $M$ with new scalar multiplication $c \cdot x=J(c) x$. Denote by ${ }_{(1+J)}$ Pic $(C)$ the subgroup of all $(M)$ in Pic $(C)$ with $(M)(\widehat{M})=$ 1. The "forgetful map" $(E, \Phi) \mapsto(E)$ from $S_{1}(C, J)$ to Pic $(C)$ yields an exact sequence

$$
1 \longrightarrow A^{*} / N C^{*} \longrightarrow S_{1}(C, J) \longrightarrow{ }_{(1+J)} \operatorname{Pic}(C) \longrightarrow 1 \text {. }
$$

(The surjectivity on the right can easily be proved, cf. [12, §2.3]).

REMARK 3.2. Let $C$ be an integral domain with quotient field $L$. 
Any element of Pic $(C)$ can be represented by an invertible ideal a and $\hat{a}$ is isomorphic with the ideal $\bar{a}=J(\mathfrak{a})$. For any hermitian from $\Phi$ on $a$ we have $\Phi(x, y)=d x \bar{y}$ for some $d$ in $L$ such that $d a \bar{a} \subset C$, since $\Phi$ is the restriction of an $(L, J)$-form on $L$. Note that $\Phi$ is non degenerate if and only if $d a \bar{a}=C$.

Now let $C$ be connected. For any $(C, J)$-space $(E, \Phi)$ of rank $n$ we define the determinant, $\operatorname{det}(E)$, of $(E, \Phi)$ to be the element of $S_{1}(C, J)$ represented by the space $\left(\Lambda^{n} E, \Delta\right)$ with $\Delta$ defined by

$$
\Delta\left(x_{1} \wedge \cdots \wedge x_{n}, y_{1} \wedge \cdots \wedge y_{n}\right)=\operatorname{det}\left(\Phi\left(x_{i}, y_{j}\right)\right),
$$

which is again a nondegenerate space [3, §2]. The map

$$
\operatorname{det}: S(C, J) \longrightarrow S_{1}(C, J)
$$

is additive but it does not vanish on all hyperbolic spaces. Thus we have to modify this map in the following well known way: denote by $\boldsymbol{Z} / 2 Z \cdot S_{1}(C, J)$ the set $\boldsymbol{Z} / 2 \boldsymbol{Z} \times S_{1}(C, J)$ with the twisted multiplication

$$
\left(u_{1}, x_{1}\right)\left(u_{2}, x_{2}\right)=\left(u_{1}+u_{2},(-1)^{u_{1} u_{2}} x_{1} x_{2}\right) .
$$

We have a surjective map

$$
\chi: Z \times S_{1}(C, J) \longrightarrow Z / 2 Z \cdot S_{1}(Z, J)
$$

via

$$
\chi(n, x)=\left(n \bmod 2,(-1)^{(n(n-1) / 2)} x\right) \text {. }
$$

It is easy to verify that $\chi$ is multiplicative so that, in particular, $\boldsymbol{Z} / 2 \boldsymbol{Z} \cdot S_{1}(C, J)$ is a group. We now consider the additive map

$$
(\nu, d): S(C, J) \stackrel{\text { (rank, det) }}{\longrightarrow} Z \times S_{1}(C, J) \stackrel{\chi}{\longrightarrow} Z / 2 Z \cdot S_{1}(C, J),
$$

i.e. $(\nu(E), d(E))=\left(n \bmod 2,(-1)^{(n(n-1)) / 2} \operatorname{det} E\right)$ for any $(E)$ in $S(C, J)$ of rank $n$, and call $d(E)$ the signed determinant of $E$. Since $(\nu, d)$ vanishes on all hyperbolic spaces [12, Satz 4.1.2, p. 108], it induces maps from $K(C, J)$ and $W(C, J)$ to $Z / 2 Z \cdot S_{1}(C, J)$ which we will also denote by $(\nu, d)$. Since for $(E)$ in $S_{1}(C, J)$ we have $d(E)=(E)$ it follows that the canonical maps $S_{1}(C, J) \rightarrow K(C, J)$ and $S_{1}(C, J) \rightarrow$ $W(C, J)$ are injective. Thus we may regard $S_{1}(C, J)$ as a subgroup of the units of $K(C, J)$ or of $W(C, J)$.

THEOREM 3.3. Let $C$ be either a Dedekind ring or a connected semi-local ring with involution $J$ and let $T$ be either $K(C, J)$ or $W(C, J)$. Then for any unit $x$ of $T$ we have

$$
x=d(x)(1+n)
$$

with a nilpotent element $n$ in $T$. In particular, the group $T^{*}$ is 
generated by $S_{1}(C, J)$ and $1+\operatorname{Nil} T$.

Proof. If $T=T_{t}$ then by Proposition 1.2 (iii) and Lemma 2.1 (iv), $M_{0, T}=$ Nil $T$. Since $x$ is a unit, it must be represented by a form of odd rank. Hence $x-d(x) \in M_{0, T}$ is nilpotent, so setting

$$
n=d(x)^{-1}(x-d(x))
$$

we have $x=d(x)(1+n)$ with $n$ nilpotent.

If $T \neq T_{t}$ then $Z \rightarrow T$ is injective so by Proposition 1.2 (iii) and Remark 2.2, Nil $T$ is the set of $y$ in $T$ such that $\sigma(y)=0$ for all ring homomorphisms $\sigma: T \rightarrow Z$. Thus we only need to check that $\sigma(x)=$ $\sigma(d(x))$ for all $\sigma$. This has been done in [14], proof of Theorem 3.23, for $C$ connected semi-local. Now let $C$ be a Dedekind ring, $L$ its quotient field, and $R$ be $K(L, J)$ or respectively $W(L, J)$. Denote the canonical map $T \rightarrow R$ by $\varphi$. By Proposition 1.2 (iii) and Remark 2.2 any homomorphism $\sigma: T \rightarrow \boldsymbol{Z}$ can be factored $\sigma=\sigma^{\prime} \circ \varphi$ with some $\sigma^{\prime}: R \rightarrow Z$. Since $\varphi(x)$ is a unit of $R$ we know from the preceding that $\sigma^{\prime}(\varphi(x))=\sigma^{\prime}(d(\varphi(x)))$. But by the functorial properties of exterior powers clearly $d(\varphi(x))=\varphi(d(x))$. Thus we get the desired equation

$$
\left(\sigma^{\prime} \circ \varphi\right)(x)=\left(\sigma^{\prime} \circ \varphi\right)(d(x)) \text {. }
$$

REMARK 3.4. Let $q$ be any rational prime, $R=Z[G] / K$ a Witt ring for an abelian group $G$ of exponent $q$ and $T$ be the intersection of the subrings $T_{i}=Z\left[H_{i}\right] /\left(Z\left[H_{i}\right] \cap K\right)$ corresponding to some family $\left\{H_{i}\right\}$ of subgroups of $G$. It can be shown that any unit of $T$ has the form $\pm \bar{g}(1+n)$ where $\bar{g}$ is the image of some $g$ in $\bigcap_{i} H_{i}$ and $n$ is in Nil $T$, in the following cases: $\left(I_{G}=\right.$ augmentation ideal).

(i) $q \neq 2: K \subset I_{G}^{2}+q Z[G]$

(ii) $q=2: K \subset I_{G}^{2}+4 Z[G]$

(iii) $q=2$ : there exists an element $w$ of $\cap H_{i}$ such that

$$
1+w \in K \subset I_{G}^{2}+4 Z[G]+(1+w) Z[G] \text {. }
$$

Note that for $R=W(L, J), G=F^{*} / N L^{*}, T=W(C, J)$ condition (ii) is violated but (iii) holds with $w=(-1)$. The assumptions (i)(iii) are needed to construct "determinant functions" playing a role similar to that of the signed determinant in the proof of Theorem 3.2.

4. $\Lambda(C, J)$ for $J \neq$ identity. If $J$ is the identity the kernel $\Lambda(C, J)$ of the canonical map $K(C, J) \rightarrow K(L, J)$ has been determined for any Dedekind ring in [9, Cor. to Th. 4] and $[12,11.3 .5$, p. 138]. In this section we give a computation of $\Lambda(C, J)$ for $J \neq$ identity. We argue along the same lines as in [12] and use well-known classical methods ([2], [15]). 
Throughout this section we use the notations of $\S 1$ : $C$ is an arbitrary Dedekind ring with an involution $J \neq$ identity, whose fixed ring is denoted by $A$. For any prime ideal $\mathfrak{p}$ of $A$, we denote by $\hat{A}_{p}$ the completion of the localization $A_{p}$, and we write $\hat{C}_{p}$ for $C \otimes_{A} \hat{A}_{p}$, the completion of $C \otimes_{A} A_{\mathfrak{p}}$ with respect to $\mathfrak{p}$. We set $\widehat{C}_{\mathfrak{p}}=L$ for $\mathfrak{p}=$ 0 and for a maximal ideal $\mathfrak{p}$ of $A$ we write $\hat{L}_{\mathfrak{p}}$ for the completion $L \otimes_{F} \hat{F}_{p}=C \otimes_{A} \hat{F}_{\mathfrak{p}}$ of $L$ which is the total ring of quotients of the semi-local ring $\hat{C}_{p}$. The involutions induced by $J$ on $\hat{C}_{p}$ and $\hat{L}_{p}$ will both be written as $\hat{J}_{p}$. For any space $E$ over $(C, J)$ we denote by $\hat{E}_{p}$ the completed localization $E \otimes_{A} \hat{A}_{p}$ which is a space over $\left(\widehat{C}_{p}, \hat{J}_{p}\right)$. As usual we consider $\hat{E}_{p}$ as a subset of the space $E \otimes_{A} \hat{F}_{\mathfrak{p}}$ over $\left(\hat{L}_{\mathfrak{p}}, \hat{J}_{\mathfrak{p}}\right)$ and the automorphism group $\mathfrak{U}\left(\hat{E}_{\mathfrak{p}}\right)$ as a subgroup of $\mathfrak{U}\left(E \otimes_{A} \hat{F}_{\mathfrak{p}}\right)$. For a space $M$ over $(L, J)$ we denote by $\hat{M}_{\mathfrak{p}}$ the completion with respect to $\mathfrak{p}$, which is a space over $\left(\hat{L}_{\mathfrak{p}}, \widehat{J}_{\mathfrak{p}}\right)$. Note that $\widehat{M}_{\mathfrak{p}}=M$ if $\mathfrak{p}=0$.

We define the genus, $\Gamma(E)$, of a space $E$ over $(C, J)$ as the set of isometry classes $\left(E^{\prime}\right)$ in $S(C, J)$ such that $\hat{E}_{\mathfrak{p}} \cong \hat{E}_{\mathfrak{p}}^{\prime}$ for all prime ideals $\mathfrak{p}$ of $A$. If $\left(E^{\prime}\right)$ lies in $\Gamma(E)$ we can find an isometry $\sigma: E \otimes L \rightarrow$ $E^{\prime} \otimes L$ and for every $\mathfrak{p}$ in $\operatorname{Max} A$ an element $\sigma_{\mathfrak{p}}$ in $\mathfrak{U}\left(E^{\prime} \otimes \hat{L}_{\mathfrak{p}}\right)$ such that

$$
\hat{E}_{p}^{\prime}=\sigma_{p} \sigma \hat{E}_{p}
$$

Since $\sigma_{\mathfrak{p}}$ is unitary, the determinant of $\sigma_{\mathfrak{p}}$ over $\hat{L}_{\mathfrak{p}}$ has norm 1 and hence by Hilbert's Theorem 90 ,

$$
\operatorname{det} \sigma_{\mathfrak{p}}=a_{\mathfrak{p}} \bar{a}_{\mathfrak{p}}^{-1}
$$

for some $a_{\mathfrak{p}}$ in $\hat{L}_{p}^{*}$. We use (4.2) to define for any genus $\Gamma$ a map

$$
\varphi: \Gamma \times \Gamma \longrightarrow \operatorname{Div} C / \mathscr{S}(\operatorname{Div} C)^{G}
$$

where Div $C$ denotes the divisor group of $C$, (Div $C)^{G}$ the subgroup left fixed by $G=\{1, J\}$ and $\mathfrak{S}$ the group of principal divisors of $C$. For $E, E^{\prime}$ in $\Gamma$ we define $\varphi\left(E, E^{\prime}\right)$ to be the image of

$$
\prod_{\mathfrak{p}} \prod_{\mathfrak{R} \mid \mathfrak{p}} \mathfrak{P}^{\text {ord }{ }_{\mathfrak{p}}\left(a_{\mathfrak{p}}\right)} \text {, }
$$

$\mathfrak{P}$ in $\operatorname{Max} C$

in $\operatorname{Div} C / \mathscr{S}(\operatorname{Div} C)^{G}$ with $a_{\mathfrak{p}}$ coming from (4.1) and (4.2) chosen arbitrarily. Here $\mathfrak{p}$ runs only through the ideals of $\operatorname{Max} A$ which split in $C$. Next, we show that $\varphi\left(E, E^{\prime}\right)$ is well defined, i.e., that (4.3) does not depend on the particular choices in (4.1) and (4.2). Given (4.1), the $a_{\mathfrak{p}}$ in (4.2) can only be changed by a factor in $\hat{F}_{\mathfrak{p}}^{*}$ which does not affect (4.3) $\bmod (\mathrm{Dw})$. Let $\hat{E}_{\mathfrak{p}}^{\prime}=\sigma_{\mathfrak{p}}^{\prime} \sigma^{\prime} E$ be another system of equations of type (4.1). Then there exists some $\rho$ in $\mathfrak{U}\left(E^{\prime} \otimes L\right)$ and $\mu_{p}$ in $\mathfrak{U}\left(\hat{E}_{\mathfrak{p}}^{\prime}\right)$ such that 


$$
\sigma_{\mathfrak{p}}^{\prime}=\mu_{\mathfrak{p}} \sigma_{\mathfrak{p}} \rho
$$

for all $\mathfrak{p}$ in $\operatorname{Max} A$. If $\mathfrak{p}$ splits in $C$ then $\hat{L}_{\mathfrak{p}} \cong \hat{F}_{\mathfrak{p}} \times \hat{F}_{\mathfrak{p}}$ and the involution $\hat{J}_{\mathfrak{p}}$ is given by $\hat{J}_{\mathfrak{p}}(x, y)=(y, x)$ [14, Lemma 1.8]. Hence the element $\operatorname{det}\left(\mu_{p}\right)$ of $\hat{C}_{p}^{*}$ of norm 1 can be written in the form $b_{p} \bar{b}_{p}^{-1}$ with $b_{\mathfrak{p}}$ in $\hat{C}_{\mathfrak{p}}^{*}$. Again by Hilbert's Theorem 90 , there is an element $c$ in $L$ such that $\operatorname{det} \rho=c \bar{c}^{-1}$. Thus starting from (4.4), we obtain for the computation of $\varphi\left(E, E^{\prime}\right)$, elements $a_{p}^{\prime}=b_{p} a_{p} c$ at split prime ideals and this yieldst he same value in (4.3) $\bmod \mathfrak{H}$. Hence it is also clear that $\varphi\left(E, E^{\prime}\right)$ depends only on the isometry classes of $E$ and $E^{\prime}$. Clearly for spaces $V, V^{\prime}$ in some other genus we have

$$
\begin{aligned}
\varphi\left(E \perp V, E^{\prime} \perp V^{\prime}\right) & =\varphi\left(E, E^{\prime}\right) \varphi\left(V, V^{\prime}\right) \text { and also } \\
\varphi\left(E, E^{\prime}\right) & =\varphi\left(E^{\prime}, E\right)^{-1}
\end{aligned}
$$

EXAmple 4.6. Let $(E, \Phi)$ be the hyperbolic space of rank 2 over $(L, J)$, i.e., $E=C e \oplus C f$ with $\Phi(e, e)=\Phi(f, f)=0, \Phi(e, f)=1$. For any $a$ in $\operatorname{Div} C$ we regard $H(\mathfrak{a})$ as the sublattice ae $\oplus \bar{a}^{-1} f$ of $E$. Choose for every $\mathfrak{p}$ in $\operatorname{Max} A$ a generator $a_{\mathfrak{p}}$ of the principal ideal $a \widehat{C}_{p}$. Then the automorphism $e \mapsto a_{\mathfrak{p}} e, f \mapsto \bar{a}_{\mathfrak{p}}^{-1} f$ of $\hat{E}_{\mathfrak{p}} \operatorname{maps} H(\hat{C})_{\mathfrak{p}}$ to $\hat{H}(\mathfrak{a})_{\mathfrak{p}}$. Thus $H(\mathfrak{a})$ and $H$ are in the same genus and $\varphi(H, H(\mathfrak{a}))=[\mathfrak{a}]$, where [a] denotes the class of the divisor a in $\operatorname{Div} C / \mathscr{S}(\operatorname{Div} C)^{G}$.

We define the $S U$-genus $\Sigma(E)$ of a space $E$ over $(C, J)$ as the set of all isometry classes $\left(E^{\prime}\right)$ in $\Gamma(E)$ such that there exists an equation (4.1) with $\sigma_{\mathfrak{p}}$ in $\operatorname{Su}\left(\hat{E}_{\mathfrak{p}}^{\prime}\right)$ for all $\mathfrak{p}$ in $\operatorname{Max} A$, i.e., with $\operatorname{det} \sigma_{\mathfrak{p}}=1$.

LEMma 4.7. Let $E$ be a $(C, J)$-space and assume that $\hat{E}_{\mathrm{p}}$ represents a unit of $\hat{A}_{\mathfrak{p}}$ for every $\mathfrak{p}$ in $\operatorname{Max} A$. (This hypothesis is always fulfilled if $\mathfrak{p}$ splits or if 2 does not lie in $\mathfrak{p}$.$) Then \Sigma(E)$ is the set of all $\left(E^{\prime}\right)$ in $\Gamma(E)$ with $\varphi\left(E, E^{\prime}\right)=1$.

Proof. If $\left(E^{\prime}\right)$ lies in $\Sigma(E)$ it is clear that $\varphi\left(E, E^{\prime}\right)=1$. Let now $\left(E^{\prime}\right)$ be in $\Gamma(E)$ with $\varphi\left(E, E^{\prime}\right)=1$. Without loss of generality we assume that $E$ and $E^{\prime}$ are lattices of full rank in the same space $M$ over $(L, J)$. First, for every $\mathfrak{p}$ in $\operatorname{Max} A$, we choose some $\sigma_{\mathfrak{p}}$ in $\mathfrak{U}\left(\widehat{M}_{\mathfrak{p}}\right)$ and some $a_{\mathfrak{p}}$ in $\hat{L}_{\mathfrak{p}}^{*}$ such that $\hat{E}_{\mathfrak{p}}^{\prime}=\sigma_{\mathfrak{p}} \hat{E}_{\mathfrak{p}}$ with $\operatorname{det} \sigma_{\mathfrak{p}}=a_{\mathfrak{p}} \bar{a}_{\mathfrak{p}}^{-1}$. We want $\operatorname{det} \sigma_{\mathfrak{p}}$ to be a unit in $\hat{C}_{p}^{*}$. This is automatically true unless $\mathfrak{p} C=\mathfrak{P} \overline{\mathfrak{P}}$ with $\mathfrak{P} \neq \mathfrak{P}$. But since $\varphi\left(E, E^{\prime}\right)=1$ we can find a $c$ in $L^{*}$ such that $\operatorname{ord}_{\mathfrak{B}}\left(a_{\mathfrak{p}} c\right)=\operatorname{ord}_{\mathfrak{B}}\left(a_{\mathfrak{p}} c\right)$ whenever $\mathfrak{p} C=\mathfrak{P} \overline{\mathfrak{P}}, \mathfrak{P} \neq \overline{\mathfrak{P}}$. Since $J \neq$ identity, $M$ contains a one-dimensional subspace $W=L e$ with $\Phi(e, e) \neq$ 0 [3, Lemma 1, p. 90 ] so that $M=L e \perp W^{\perp}$. Now let $\rho$ in $\mathfrak{u}(M)$ be the automorphism which is the identity on $W^{\perp}$ and multiplies $e$ by $c^{-1} \bar{c}$. Setting $\tau_{\mathfrak{p}}=\sigma_{\mathfrak{p}} \rho^{-1}$ we obtain

$$
\hat{E}_{\mathfrak{p}}^{\prime}=\tau_{\mathrm{p}} \rho \hat{E}_{\mathrm{p}}
$$


and $\operatorname{det} \tau_{p}=\left(\operatorname{det} \sigma_{p}\right)\left(c \bar{c}^{-1}\right)$, which is a unit of $\hat{C}_{p}$ for all $\mathfrak{p}$. By hypothesis every $\hat{E}_{\mathfrak{p}}^{\prime}$ has a subspace $\hat{C}_{p} e_{\mathfrak{p}}$ with $\Phi\left(e_{p}, e_{p}\right)$ in $A_{p}^{*}$. So by an argument as above we can find a $\mu_{p}$ in $\mathfrak{U}\left(\hat{E}_{p}^{\prime}\right)$ with $\operatorname{det} \mu_{p}=\left(\operatorname{det} \tau_{p}\right)^{-1}$. The equations

$$
\hat{E}_{\mathrm{p}}^{\prime}=\mu_{\mathrm{p}} \tau_{\mathrm{p}} \rho \hat{E}_{\mathrm{p}}
$$

show that $\left(E^{\prime}\right)$ lies in the $S U$-genus of $E$.

We now sketch a proof of a weak version of Kneser's strong approximation theorem for the special unitary group [15]. Let $(M, \Phi)$ be any space over $(L, J)$ of dimension $\geqq 2$. Then $\mathfrak{U}(M)$ is generated by the symmetries

$$
\sigma(x, l): z \longmapsto z-\Phi(z, x) l^{-1} x
$$

where $(x, l)$ runs through all pairs of $M \times L^{*}$ such that $l+\bar{l}=$ $\Phi(x, x) \neq 0[8, \mathrm{p} .41]$. We first indicate a proof for the following wellknown fact [2, §3] since we feel that the argument in [2] is not quite clear.

Lemma 4.8. Given maximal ideals $\mathfrak{p}_{1}, \cdots, \mathfrak{p}_{r}$ of $A$ and pairs $\left(x_{i}, l_{i}\right)$ in $\hat{M}_{p_{i}} \times \hat{L}_{p_{i}}^{*}, 1 \leqq i \leqq r$, with $l_{i}+\bar{l}_{i}=\Phi\left(x_{i}, x_{i}\right) \neq 0$, there exists gor every $\varepsilon>0$ a pair $(x, l)$ in $M \times L^{*}$ with $l+\bar{l}=\Phi(x, x) \neq 0$ such that $\left\|x-x_{i}\right\|_{p_{i}}<\varepsilon,\left|l-l_{i}\right|_{p_{i}}<\varepsilon$ for $1 \leqq i \leqq r$. Here ||$_{p}$ denotes $a$ valuation belonging to $\mathfrak{p}$ and \|\|$_{p}$ an associated norm on $\widehat{M}_{p}$.

Proof. Since $J \neq$ identity there is some $m$ in $L$ with $m+\bar{m}=$ 1. We can find a vector $x$ in $M$ which is near $x_{i}$ at $\mathfrak{p}_{i}$ and an $l^{\prime}$ in $L$ which is near $l_{i}$ at $\mathfrak{p}_{i}$, for $1 \leqq i \leqq r$. Then

$$
l=l^{\prime}+m\left(\Phi(x, x)-l^{\prime}-\bar{l}^{\prime}\right)
$$

satisfies $l+\bar{l}=\Phi(x, x)$ and the pair $(x, l)$ has the desired properties if the approximation of the $x_{i}$ by the $x$ and the $l_{i}$ by $l^{\prime}$ is good enough.

According [2, §3] this lemma implies that the weak approximation theorem holds for $\mathfrak{U}(M)$, i.e., the diagonal map from $\mathfrak{U}(M)$ to

$$
\prod_{p \ln S} \mathfrak{U}\left(\hat{M}_{p}\right)
$$

has dense image for any finite subset $S$ of $\operatorname{Max} A$.

Assume now that $[M: L] \geqq 3$ and that $M$ is isotropic, i.e., $M$ contains some $x \neq 0$ with $\Phi(x, x)=0$. Then $S \mathfrak{U}(M)$ is the commutator subgroup of $\mathfrak{U}(M)$ [8, Chap. II, $\S 4, \S 5]$. Thus it can be verified that the weak approximation theorem also holds for $S \mathfrak{U}(M)$. Again pick some $m$ in $L$ with $m+\bar{m}=1$. As is shown in [1] (cf. [8, p. 61] for the orthogonal case), $S \mathfrak{U}(M)$ is generated by automorphisms 


$$
E(x, y): z \longmapsto z+\Phi(z, x) y-\Phi(z, y) x-m \Phi(z, x) \Phi(y, y) x
$$

where $(x, y)$ runs through all elements of $M \times M$ with $\Phi(x, x)=$ $\Phi(x, y)=0$. Now the simple argument in [12, Proof of Satz 11.2.8, p. 137] shows that we have strong approximation in $S \mathfrak{U}(M)$. (It also seems possible to adopt the proof of [2, Satz 18]):

THEOREM 4.9. Let $M$ be an isotropic space over $(L, J)$ with ${ }^{2)}$ $[M: L] \geqq 3$ and let $E \subset M$ be a $(C, J)$-lattice of full rank. Prescribe lattices $E_{\mathfrak{p}_{i}}^{\prime}$ of full rank in $\hat{M}_{\mathfrak{p}_{i}}$ and elements $\sigma_{i}$ in $S \mathfrak{S u}\left(\hat{M}_{\mathfrak{p}_{i}}\right)$ for finitely many ideals $\mathfrak{p}_{1}, \cdots, \mathfrak{p}_{r}$ of $\operatorname{Max} A$. Then there exists an element $\sigma$ of $S \mathfrak{U}(M)$ such that $\left(\sigma-\sigma_{i}\right) \hat{E}_{\mathfrak{p}_{i}} \subset E_{\mathfrak{p}_{i}}^{\prime}$ for $1 \leqq i \leqq r$ and $\sigma \hat{E}_{\mathrm{q}}=\hat{E}_{\mathrm{q}}$ for $\mathfrak{q}$ in $\operatorname{Max} A$ distinct from $\mathfrak{p}_{1}, \cdots \mathfrak{p}_{r}$.

From this theorem a standard argument [15], [2, p. 99] establishes

COROLLARY 4.10. If $E$ is an isotropic $(C, J)$-space of rank $\geqq 3$, the $S U$-genus $\Sigma(E)$ contains only the class $(E)$.

Finally, Corollary 4.10 and Lemma 4.7 yield the desired

THEOREM 4.11. The kernel of the surjection $f: \operatorname{Pic}(C) \rightarrow \Lambda(C, J)$, where $f(\mathfrak{a})=[H(\mathfrak{a})]-[H]$, is the subgroup $\mathfrak{E}(\operatorname{Div} C)^{a} / \mathfrak{S}$.

Proof. For any $\mathfrak{a}$ in $(\operatorname{Div} C)^{G}$ we consider the spaces $E=H \perp(1)$ and $E^{\prime}=H($ a $) \perp(1)$. According to (4.5) and Example 4.6, we have $\varphi\left(E, E^{\prime}\right)=1$. Now, since $(H(\hat{a}))_{\text {p }}$ and $\hat{H}_{\mathfrak{p}}$ represent all of $A_{\mathfrak{p}}^{*}$, Lemma 4.7 shows that $E^{\prime}$ is in the $S U$-genus of $E$ and Corollary 4.10 forces $E \cong E^{\prime}$. Hence $f(\mathfrak{a})=\left[E^{\prime}\right]-[E]=0$.

Let $(\mathfrak{a})$ be in $\operatorname{Ker} f$. Then there exists a $(C, J)$-space $V$ such that $H \perp V \cong H(\mathfrak{a}) \perp V$. Again, (4.5) yields $\varphi(H, H(\mathfrak{a}))=1$ and so by Example 4.6, the class of $\mathfrak{a}$ is in $\mathfrak{S}(\operatorname{Div} C)^{G} / \mathfrak{S}$.

\section{REFERENCES}

1. R. Baeza, Unitäre Gruppen über lokalen Ringen, Dissertation, Saarbrücken, 1970.

2. S. Böge, Schiefhermitesche Formen über Zahlkörper und Quaternionenschiefkörpern, J. reine angew. Math., 221 (1966), 85-112.

3. N. Bourbaki, Algèbre, Chap. 9, Act. Sc. Ind. 1272, Hermann, Paris 1959.

4. — Algèbre commutative, Chap. 1-2, Act. Sc. Ind. 1290, Hemann, Paris 1961.

5. —, Algèbre commutative, Chap. 5, Act. Sc. Ind. 1308, Hermann, Paris 1964.

6. - Algèbre commutative, Chap. 7, Act. Sc. Ind. 1314, Hermann, Paris 1965.

7. H. Cartan and S. Eilenberg, Homological algebra, Princeton University Press, Princeton 1956.

${ }^{2}$ The condition $[M: L] \geqq 3$ has inadvertently been omitted in [2, 11.2]. 
๑. J. Dieudonné, La géométrie des groupes classiques, 2nd Ed., Ergebnisse d. Math., Springer, Berlin 1963.

9. A. Fröhlich, On the K-theory of unimodular forms over rings of algebraic integers, Quar. J. Math., (Oxford), 22 (1971), 401-423.

10. D. K. Harrison, Witt rings, Lecture notes, Department of Mathematics, University of Kentucky, Lexington, Kentucky, 1970.

11. I. Kaplansky, Commutative rings, Allyn and Bacon, Boston, 1970.

12. M. Knebusch, Grothendieck und Wittringe von nichtausgearteten symmetrischen Bilinearformen, Sitzber. Heidelberg Akad. Wiss. (1969/1970), 93-157.

13. M. Knebusch, A. Rosenberg and R. Ware, Structure of Witt rings, quotients of abelian group rings, and orderings of fields, Bull. Amer. Math. Soc., 77 (1971), 205-210. 14. - Structure of Witt rings and quotients of abelian group rings, Amer. J. Math., 94 (1972), 119-155.

15. M. Kneser, Klassenzahlen indefiniter quadratischer Formen in drei und mehr Veränderlichen, Archiv Math., 7 (1956), 323-332.

16. J. Leicht und F. Lorenz, Die Primideale des Wittschen Ringes, Invent. Math., 10 (1970), 82-88.

17. J. Milnor, Symmetric inner product spaces in characteristic two, in Ann. of Math. Studies 70, Princeton University Press, Princeton, New Jersey, 1971.

18. A. Pfister, Quadratische Formen in beliebigen Körpern, Invent. Math., 1 (1966), $116-132$.

19. W. Scharlau, Klassifikation hermitescher Formen über lokalen Körpern, Math. Ann., 186 (1970), 201-208.

20. J. Serre, Modules projectifs et éspaces fibrés à fibre vectorielle, Sém. Dubreil-Pisot, Paris 1957-58.

21. T. A. Springer, Quadratic forms over fields with a discrete valuation, Indag. Math., 17 (1955), 352-362.

22. E. Steinitz, Rechteckige Systeme und Moduln in algebraischen Zahlkörpern, Math. Ann., 71 (1912), 328-354.

Received July 21, 1971.

UNIVERSITÄT DES SAARLANDES

CORNELL UNIVERSITY

NORTHWESTERN UNIVERSITY 



\section{PACIFIC JOURNAL OF MATHEMATICS}

\section{EDITORS}

\author{
H. SAMELSON \\ Stanford University \\ Stanford, California 94305 \\ C. R. HOBBY \\ University of Washington \\ Seattle, Washington 98105
}

\section{J. DugundjI}

Department of Mathematics University of Southern California Los Angeles, California 90007

\section{RICHARD ARENS}

University of California

Los Angeles, California 90024

\section{ASSOCIATE EDITORS}
E. F. BECKENBACH
B. H. NeumanN
F. WOLF
K. YosHIDA

\section{SUPPORTING INSTITUTIONS}

\author{
UNIVERSITY OF BRITISH COLUMBIA \\ CALIFORNIA INSTITUTE OF TECHNOLOGY \\ UNIVERSITY OF CALIFORNIA \\ MONTANA STATE UNIVERSITY \\ UNIVERSITY OF NEVADA \\ NEW MEXICO STATE UNIVERSITY \\ OREGON STATE UNIVERSITY \\ UNIVERSITY OF OREGON \\ OSAKA UNIVERSITY
}

\author{
UNIVERSITY OF SOUTHERN CALIFORNIA \\ STANFORD UNIVERSITY \\ UNIVERSITY OF TOKYO \\ UNIVERSITY OF UTAH \\ WASHINGTON STATE UNIVERSITY \\ UNIVERSITY OF WASHINGTON \\ ${ }^{*} \stackrel{*}{*}{ }^{*}$
AMERICAN MATHEMATICAL SOCIETY
NAVAL WEAPONS CENTER
}

The Supporting Institutions listed above contribute to the cost of publication of this Journal, but they are not owners or publishers and have no responsibility for its content or policies.

Mathematical papers intended for publication in the Pacific Journal of Mathematics should be in typed form or offset-reproduced, (not dittoed), double spaced with large margins. Underline Greek letters in red, German in green, and script in blue. The first paragraph or two must be capable of being used separately as a synopsis of the entire paper. The editorial "we" must not be used in the synopsis, and items of the bibliography should not be cited there unless absolutely necessary, in which case they must be identified by author and Journal, rather than by item number. Manuscripts, in duplicate if possible, may be sent to any one of the four editors. Please classify according to the scheme of Math. Rev. Index to Vol. 39. All other communications to the editors should be addressed to the managing editor, Richard Arens, University of California, Los Angeles, California, 90024.

50 reprints are provided free for each article; additional copies may be obtained at cost in multiples of 50 .

The Pacific Journal of Mathematics is published monthly. Effective with Volume 16 the price per volume (3 numbers) is $\$ 8.00$; single issues, $\$ 3.00$. Special price for current issues to individual faculty members of supporting institutions and to individual members of the American Mathematical Society: $\$ 4.00$ per volume; single issues $\$ 1.50$. Back numbers are available.

Subscriptions, orders for back numbers, and changes of address should be sent to Pacific Journal of Mathematics, 103 Highland Boulevard, Berke'ey, California, 94708.

PUBLISHED BY PACIFIC JOURNAL OF MATHEMATICS, A NON-PROFIT CORPORATION

Printed at Kokusai Bunken Insatsusha (International Academic Printing Co., Ltd.), 270, 3-chome Totsuka-cho, Shinjuku-ku, Tokyo 160, Japan. 


\section{Pacific Journal of Mathematics}

\section{Vol. 43, No. $3 \quad$ May, 1972}

Max K. Agoston, An obstruction to finding a fixed point free map on a manifold.... 543

Nadim A. Assad and William A. Kirk, Fixed point theorems for set-valued mappings

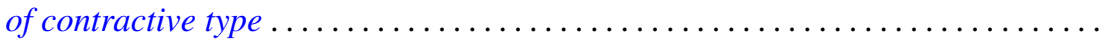

John Winston Bunce, Characterizations of amenable and strongly amenable

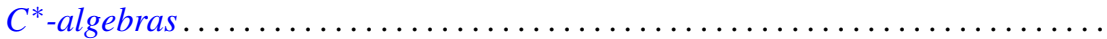

Erik Maurice Ellentuck and Alfred Berry Manaster, The decidability of a class of

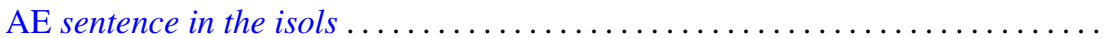

U. Haussmann, The inversion theorem and Plancherel's theorem in a Banach

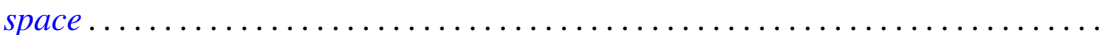

Peter Lawrence Falb and U. Haussmann, Bochner's theorem in infinite dimensions.

Peter Fletcher and William Lindgren, Quasi-uniformities with a transitive base ..... Dennis Garbanati and Robert Charles Thompson, Classes of unimodular abelian

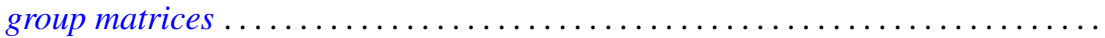

Kenneth Hardy and R. Grant Woods, On c-realcompact spaces and locally bounded normal functions

Manfred Knebusch, Alex I. Rosenberg and Roger P. Ware, Grothendieck and Witt rings of hermitian forms over Dedekind rings .......................

George M. Lewis, Cut loci of points at infinity.

Jerome Irving Malitz and William Nelson Reinhardt, A complete countable $L_{\omega_{1}}^{Q}$ theory with maximal models of many cardinalities . . . . . . . . . . . . . . . . .

Wilfred Dennis Pepe and William P. Ziemer, Slices, multiplicity, and Lebesgue

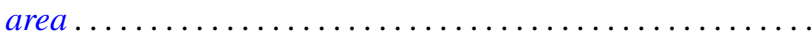

Keith Pierce, Amalgamating abelian ordered groups . .

Stephen James Pride, Residual properties of free groups . . . . . . . . . . . . . 725

Roy Martin Rakestraw, The convex cone of n-monotone functions .

T. Schwartzbauer, Entropy and approximation of measure preserving transformations .

Peter F. Stebe, Invariant functions of an iterative process for maximization of a polynomial...

Kondagunta Sundaresan and Wojbor Woyczynski, L-orthogonally scattered

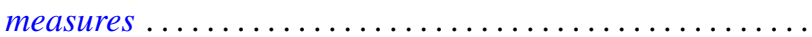

Kyle David Wallace, $C_{\lambda}$-groups and $\lambda$-basic subgroups $\ldots \ldots \ldots$

Barnet Mordecai Weinstock, Approximation by holomorphic functions on certain

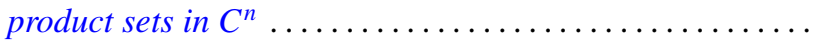

Donald Steven Passman, Corrections to: "Isomorphic groups and group rings”.

Don David Porter, Correction to: "Symplectic bordism, Stiefel-Whitney numbers, and a Novikov resolution"

John Ben Butler, Jr., Correction to: “Almost smooth perturbations of self-adjoint operators".

Constantine G. Lascarides, Correction to: "A study of certain sequence spaces of Maddox and a generalization of a theorem of Iyer" ...... ...

George A. Elliott, Correction to: "An extension of some results of takesaki in the reduction theory of von neumann algebras" ......................... 